\title{
Modest overall survival improvements from 1998 to 2009 in metastatic gastric cancer patients: a population-based SEER analysis
}

\author{
Sabrina M. Ebinger ${ }^{1} \cdot$ René Warschkow $^{1,2} \cdot$ Ignazio Tarantino $^{3} \cdot$ Bruno M. Schmied $^{1} \cdot$ \\ Ulrich Güller $^{4} \cdot$ Marc Schiesser $^{1}$
}

Received: 20 May 2015/ Accepted: 1 September 2015/Published online: 21 September 2015

(c) The International Gastric Cancer Association and The Japanese Gastric Cancer Association 2015

\begin{abstract}
Background An increasing fraction of gastric cancer patients present with distant metastases at diagnosis. The objective of the present 11-year population-based trend analysis was to assess the survival rates in patients who underwent and in patients who did not undergo palliative gastrectomy.

Methods Patients with metastatic gastric cancer were identified from the Surveillance, Epidemiology, and End Results (SEER) database between 1998 and 2009. Time trend and impact of palliative gastrectomy on survival were assessed by both a multivariate Cox proportional hazards model and propensity score matching.

Results We identified 8249 patients with stage IV gastric cancer. The rate of metastatic disease increased from $31.0 \%$ in 1998 to $37.5 \%$ in $2009(P<0.001)$. The palliative gastrectomy rate dropped from 18.8 to $10.2 \%$ $(P=0.004)$. The median survival for patients who underwent palliative gastrectomy $(N=1445,17.4 \%)$ and for patients who did not undergo palliative gastrectomy $(N=6804,82.4 \%)$ was 7 and 3 months, respectively.
\end{abstract}

S. M. Ebinger and R. Warschkow contributed equally.

Marc Schiesser

marc.schiesser@kssg.ch

1 Department of Surgery, Cantonal Hospital of St. Gallen, 9007 St Gallen, Switzerland

2 Institute of Medical Biometry and Informatics, University of Heidelberg, 69120 Heidelberg, Germany

3 Department of Surgery, University of Heidelberg, 69120 Heidelberg, Germany

4 Department of Medical Oncology and Haematology, Cantonal Hospital of St Gallen, 9007 St Gallen, Switzerland
There was an increase in median overall survival from 2 months (1998) to 3 months (2009) in the no-gastrectomy group, and from 6.5 to 8 months in the gastrectomy group. The 3-year cancer-specific survival rates were $2.1 \%$ (95\% confidence interval $1.7-2.5 \%$ ) for patients who did not undergo palliative gastrectomy and $9.4 \%$ (95\% confidence interval $7.8-11.2 \%$ ) for patients who underwent palliative gastrectomy $(P<0.001)$. Palliative gastrectomy was associated with an increased cancer-specific survival in propensity-score-adjusted Cox regression analyses (hazard ratio $0.50,95 \%$ confidence interval $0.46-0.55$, $P<0.001)$.

Conclusion On a population-based level, only modest improvements in prognosis for metastatic gastric cancer were observed in patients who underwent and in patients who did not undergo palliative gastrectomy. Considering the low rate of midterm survivors in both groups, only a small subgroup of patients benefits from palliative gastrectomy.

Keywords Metastatic gastric cancer - Palliative gastrectomy · Relative survival · Cancer specific survival · Surveillance, Epidemiology, and End Results Program

\section{Introduction}

The worldwide incidence of gastric cancer has decreased considerably during the last few decades [1]; however, gastric cancer is still associated with a poor prognosis and is the third leading cause of cancer-specific death worldwide [2]. Its case fatality rate is as high as $74 \%-$ compared with $50.4 \%$ for colorectal cancer [2] - and up to $80 \%$ of patients present with advanced disease at the time 
of the diagnosis [3]. For patients with metastatic gastric cancer the median survival is only 3-5 months without treatment and is about 10 months with palliative chemotherapy $[4,5]$ in the setting of prospective studies.

An ongoing debate is the value of palliative gastrectomy in selected patients. According to the current guidelines, gastrectomy is advocated for symptomatic tumors in the palliative setting, but not for asymptomatic tumors [6]. However, there are important arguments for the resection of the primary tumor, particularly the prevention of impending complications such as bleeding, perforation, and obstruction [7, 8]. In addition, the resection of an obstructing tumor restores the ability to swallow, and improves the nutritional status and therefore the quality of life $(\mathrm{QoL})$ [9]. Furthermore, the perioperative morbidity and mortality rates for gastrectomy have decreased substantially over the last few decades, the latter ranging from 0 to $15 \%$ [10-12], and elective palliative gastrectomy is associated with a lower complication rate compared with the emergency situation [13]. On the other hand, relevant arguments against palliative gastrectomy are that perioperative morbidity might lead to a postponed or even impeded palliative systemic therapy and also to a decreased QoL [14-16].

Whether patients benefit from palliative gastrectomy regarding survival remains contentious in the literature. Some analyses demonstrate a survival benefit $[11,17-20]$, and a recent meta-analysis of 19 nonrandomized studies showed significantly higher 1-year survival in patients undergoing gastrectomy [19]. However, undoubtedly a relevant selection bias is present since younger and healthier patients with less tumor load have a higher propensity of being operated on, whereas older, comorbid patients with multitopic metastases are less likely to undergo surgery. Some studies even concluded there is a missing or negative impact on survival [21, 22].

Therefore, the objective of this investigation was to assess trends in overall and cancer-specific survival in patients with metastatic gastric cancer in the subsets of those who underwent and those who did not undergo palliative gastrectomy on a population-based level by application of propensity score methods to minimize selection bias.

\section{Methods}

\section{Surveillance, Epidemiology, and End Results database and cohort definition}

The text data version of the Surveillance, Epidemiology, and End Results (SEER) Program of the National Cancer Institute in the USA was the data source for the present investigation [23]. Of 50,266 patients who received a diagnosis of metastatic gastric cancer between 1998 and 2009, 8249 were eligible for analysis (Fig. 1). These patients were grouped according to whether or not they have undergone gastrectomy. Patients who died before recommended surgery were included in the gastrectomy group.

\section{Statistical analysis}

Statistical analyses were performed with $\mathrm{R}$ (http://www. r-project.org). A two-sided $P$ value of less than 0.05 was considered significant. For comparing proportions and continuous variables, chi-square statistics and $t$ tests were used, respectively. For trend analysis, Spearman's rank correlation was used. Gastrectomy was assessed as a prognostic factor for survival in Cox regression analysis with and without risk adjustment for the year of diagnosis, age, ethnicity, marital status, place of birth, location of tumor, grading, and radiation therapy. For better adjustment for potential baseline confounding variables, a propensity score analysis was performed [24-26]. The MatchIt and optmatch $\mathrm{R}$ packages were used to perform a bipartite weighting propensity score analysis (full matching) with stratification for the year of diagnosis [27, 28]. Separately for each year of the study period, the cohort was partitioned into subgroups. Each subgroup contained a subset of one or more patients who underwent gastrectomy and was matched with a counterpart subset of one or more patients who did not undergo gastrectomy. In the latter subset of patients who did not undergo gastrectomy, a weight was assigned to each patient. The partitioning and weighting was performed such that the weighted average of the estimated distance measure between each patient who underwent gastrectomy and each patient who did not undergo gastrectomy within each subgroup was optimally minimized [27]. Patients who underwent gastrectomy not having a counterpart among the patients who did not undergo gastrectomy and vice versa had to be excluded from this part of the analysis. Thereafter, we assessed the prognostic value of gastrectomy in Cox regressions using the weights and strata obtained by the propensity score matching with and without adjustment for other potentially confounding variables. Finally, the putative effect of gastrectomy on survival was analyzed in another propensity score analysis based on the inverse probability of treatment weighting with use of the CBPS R package. This method is related to a marginal structural model, which incorporates all covariate balancing conditions across multiple time periods and estimates the inverse treatment probability weights to improve the resulting covariate balance [29]. 


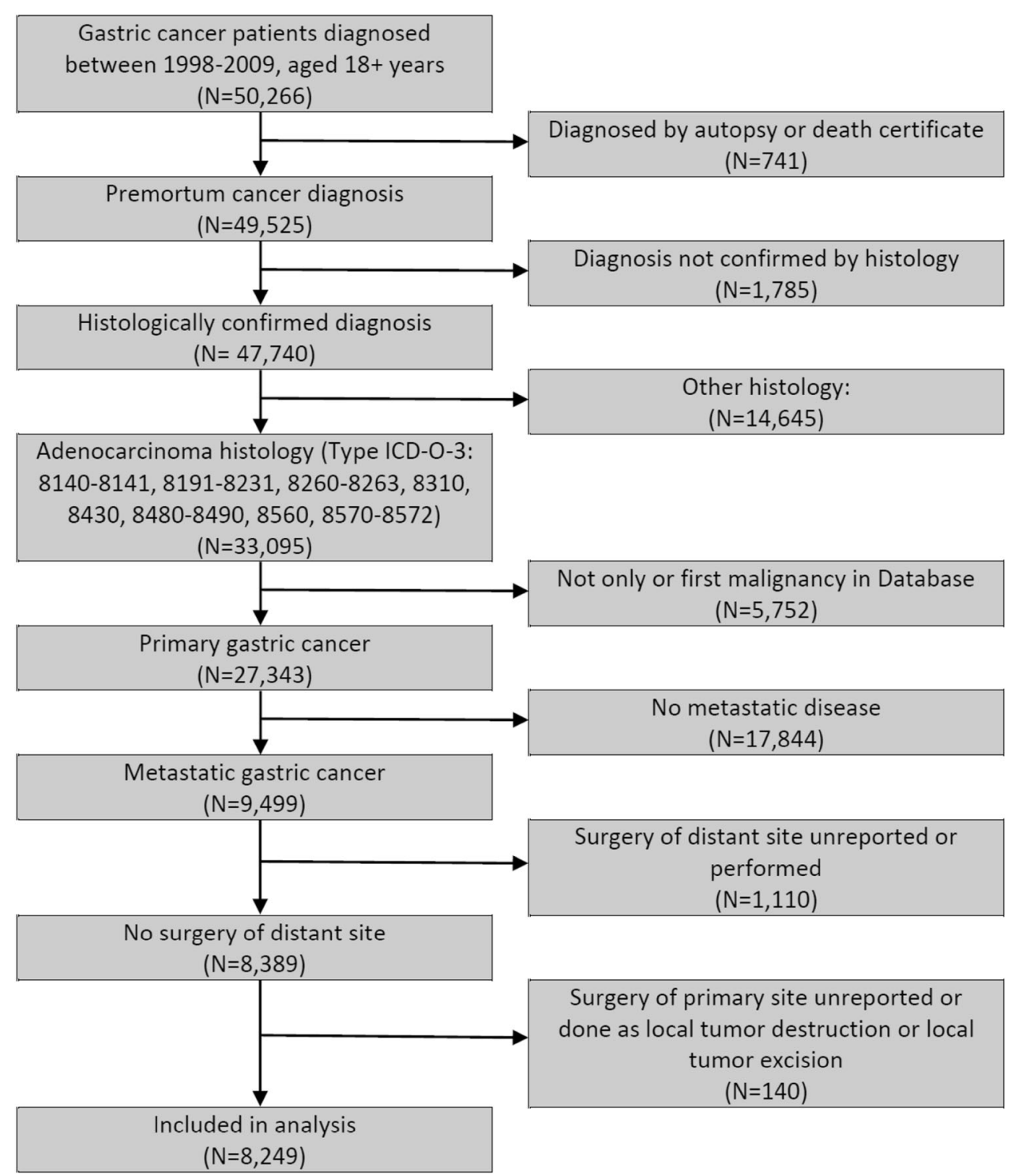

Fig. 1 Flowchart for creation of the Surveillance, Epidemiology, and End Results patient data set

\section{Results}

\section{Patient characteristics and treatment trends}

From a total of 8249 patients with metastasized gastric cancer, 1432 patients underwent gastrectomy and $6817 \mathrm{did}$ not undergo gastrectomy. Patients who died before planned surgery could be performed $(N=13)$ were included in the gastrectomy group. Hence, the analysis was based on 1445 patients in the gastrectomy group and 6804 patients in the no-gastrectomy group. Table 1 summarizes the patient characteristics for both groups and outlines the significant differences between the two groups, thus indicating a relevant bias.

The rate of metastatic disease increased significantly from $31.0 \%$ in 1998 to $37.5 \%$ in $2009(P<0.001)$.
Figure 2 displays the trends for the treatment of gastric cancer. The gastrectomy rate in patients with metastatic disease dropped from $18.8 \%$ in 1998 to $10.2 \%$ in 2009 $(P=0.004)$. This decrease occurred mainly after 2006 , when the gastrectomy rate was still $18.3 \%$. A similar drop was observed for patients younger than 65 years $(20.0 \%$ in 1998 to $10.1 \%$ in 2009) and for patients aged 65 years or more (18.1\% in 1998 to $10.3 \%$ in 2009).

\section{Gastrectomy as a prognostic factor for survival}

The 1-, 2-, and 3-year cancer-specific survival rates for patients who underwent palliative gastrectomy were $32.4 \%$ [95\% confidence interval (CI) 30.0-34.9\%], $15.9 \%(95 \%$ CI $14.0-18.0 \%)$, and $9.4 \%(95 \%$ CI $7.8-11.2 \%) \quad$ compared with $12.8 \% \quad(95 \%$ CI 


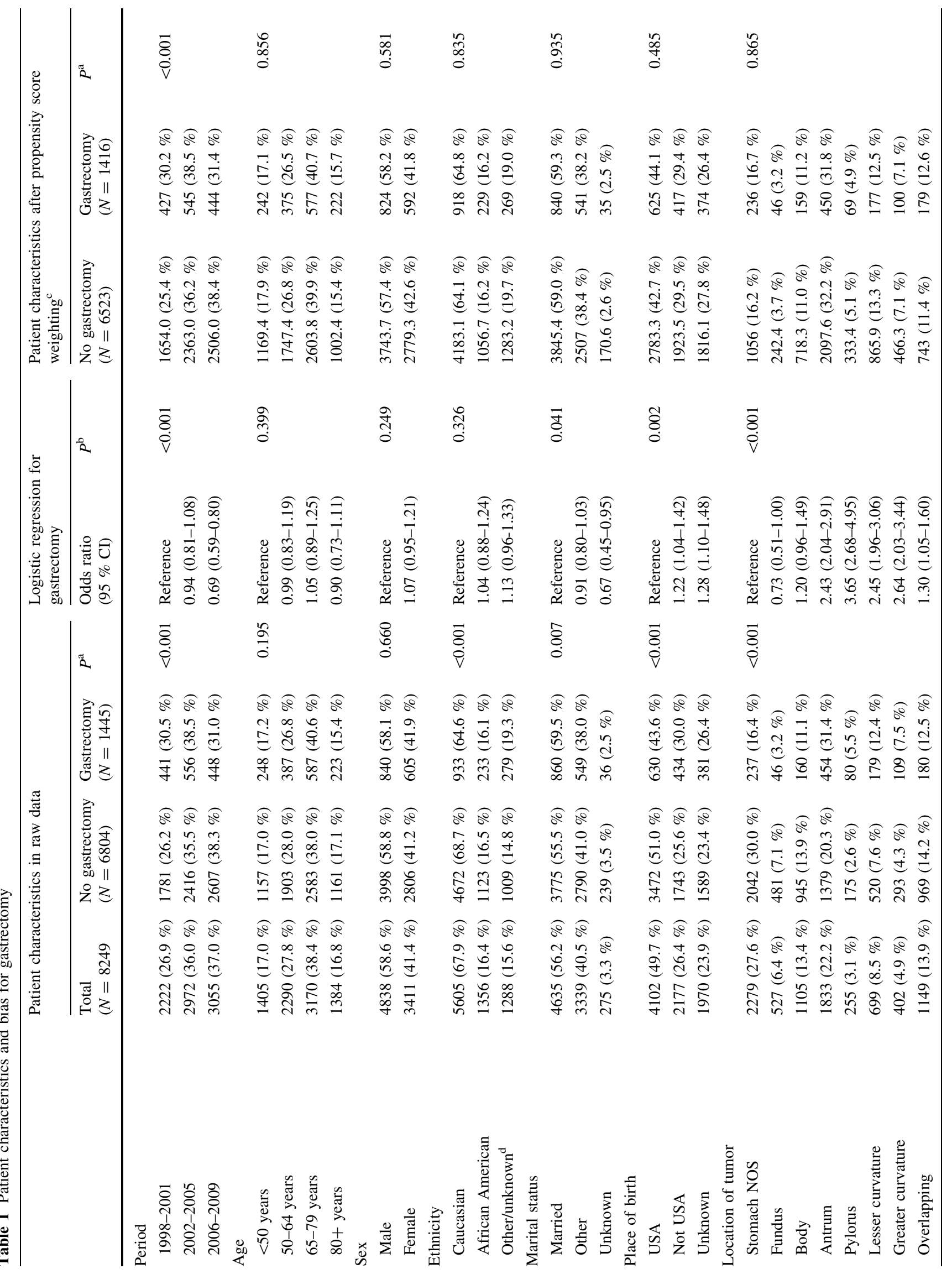




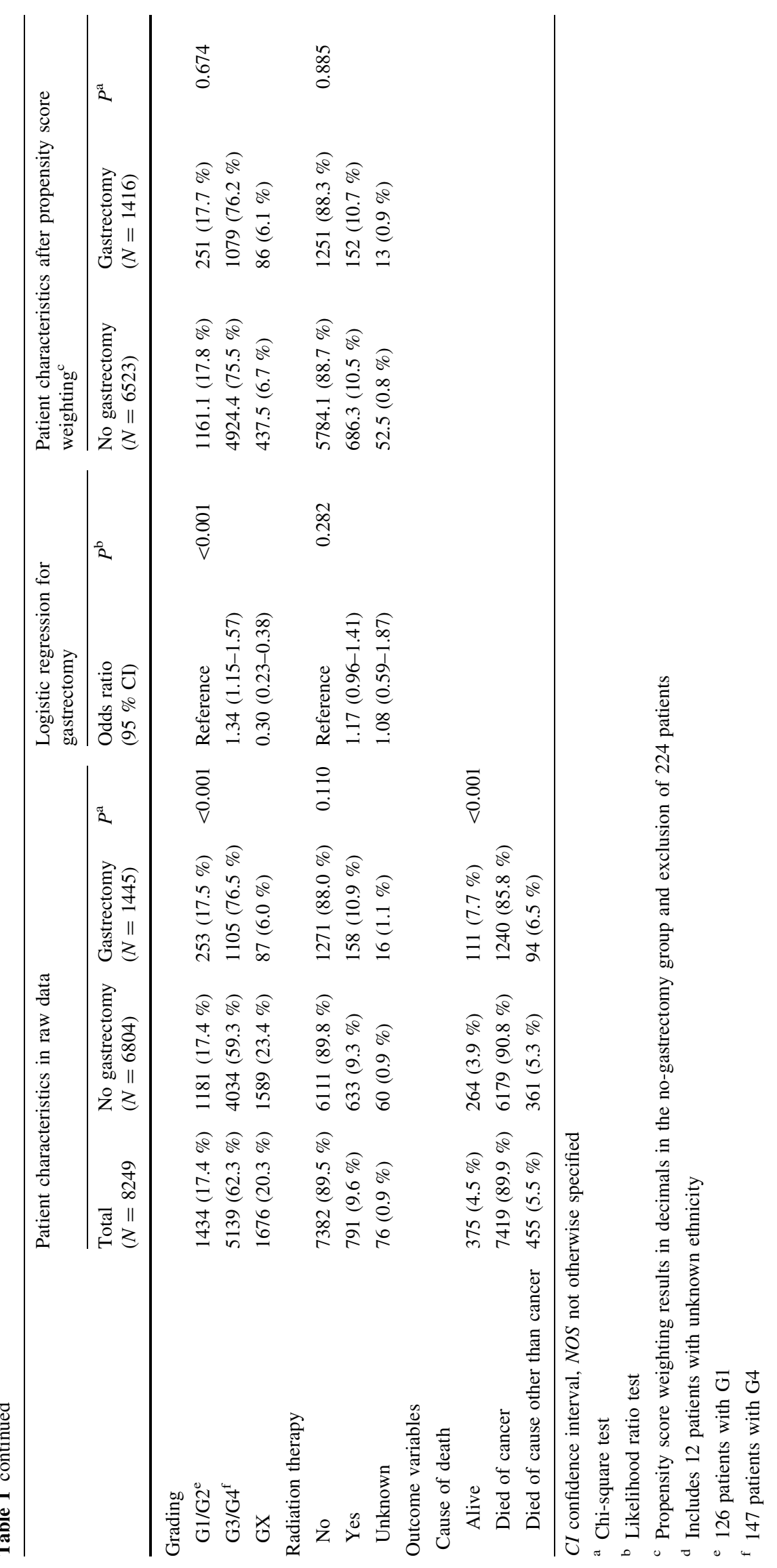




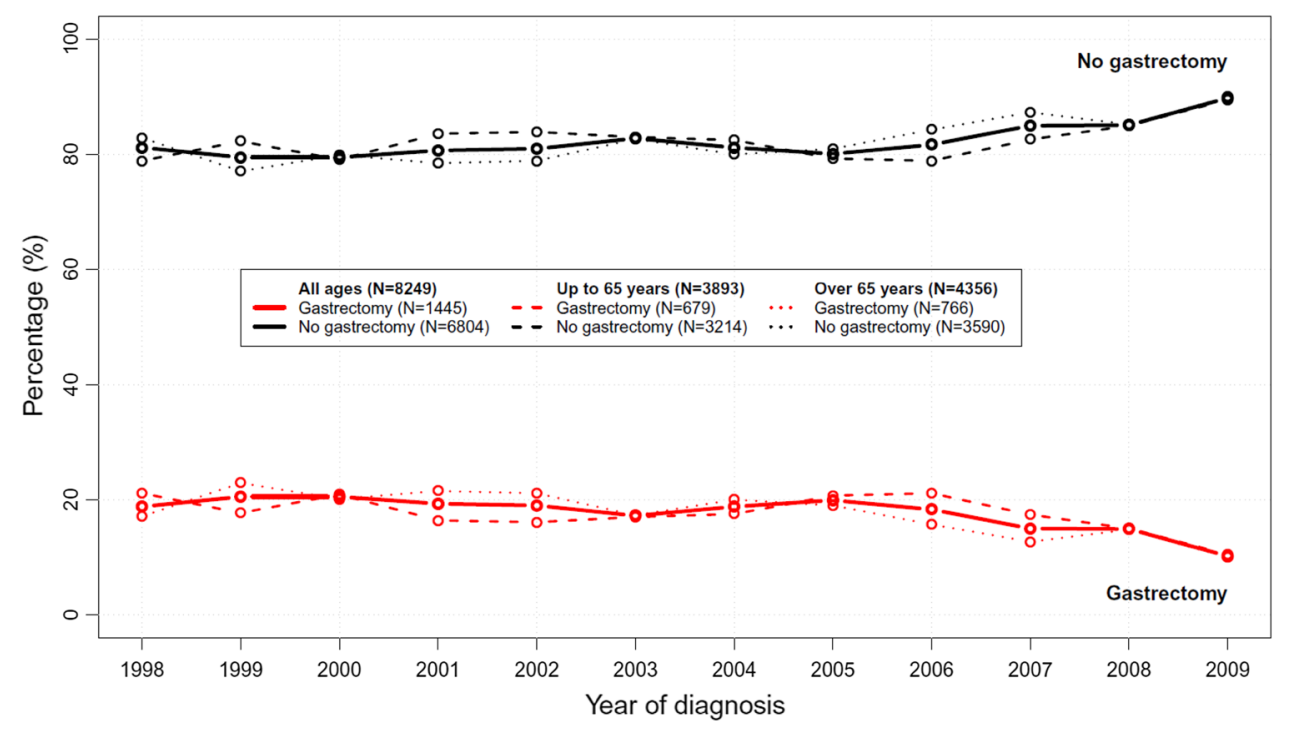

Fig. 2 Trend for treatment of metastatic gastric cancer

12.0-13.6\%), $4.4 \%$ (95\% CI 3.9-5.0\%), and $2.1 \%$ (95\% CI 1.7-2.5\%) for patients who did not undergo gastrectomy. The median survival time was 7 months compared with 3 months $(P<0.001)$. Figure 3 further elaborates the role of gastrectomy for the oncologic outcome over time. The Kaplan-Meier-curves for overall and cancer-specific survival (upper panels) demonstrate longer survival times for patients who underwent gastrectomy. When the median overall and cancer-specific survival times (middle panels) are compared, the prognosis of patients who underwent gastrectomy is generally better. For both groups a significant trend of increasing survival over time becomes evident (lower panels). These findings were confirmed by an unadjusted Cox proportional hazards regression analysis in which gastrectomy was a consistently statistically significant protective factor for overall survival [hazard ratio for death (HR) 0.55 , $95 \%$ CI 0.52-0.58, $P<0.001]$ and cancer-specific survival (HR 0.54, $95 \%$ CI 0.51-0.57, $P<0.001$ ) (Table 2). After multivariable risk adjustment of the Cox regression analysis, gastrectomy is still associated with a significantly decreased risk of overall death (HR 0.54, $95 \%$ CI $0.51-0.58, P<0.001$ ) and cancer-specific death (HR 0.53, $95 \%$ CI $0.50-0.57, P<0.001$ ) (Table 2). Figure 3 (lower panels) additionally shows the hazard ratios for overall and cancer-specific survival with multivariable adjustment for each year of diagnosis. The protective effect of gastrectomy for survival remains fairly stable during 1998-2008.

\section{Adjustment for patients characteristics with propensity score matching}

In multivariable logistic regression, patients who underwent gastrectomy received a diagnosis earlier, were married, differed in the distribution of the tumor location, and had higher grading scores (Table 1). To further adjust for this bias, a propensity score with stratification for the year of diagnosis was estimated to optimally adjust the data for the bias between the two groups. The propensity for patients who underwent gastrectomy was $0.25 \pm 0.12$ compared with $0.16 \pm 0.11$ for patients who did not undergo gastrectomy $(P<0.001)$. After we had performed the propensity score matching procedure, the score became nearly identical for patients who underwent gastrectomy and for patients who did not undergo gastrectomy $(0.25 \pm 0.12$ v. $0.25 \pm 0.12, P=0.090)$. During the propensity score matching, 292 patients (26 patients who underwent gastrectomy and 266 patients who did not undergo gastrectomy) had to be excluded because they did not have a counterpart propensity score in the other group. The three rightmost columns in Table 1 summarize the patient characteristics obtained after propensity score weighting. No significant differences between the two groups persisted after the propensity score matching and weighting except for the year of diagnosis, for which the propensity score was stratified.

\section{Propensity-score-matched prognostic factors for survival}

When we performed a Cox regression analysis using the weights and strata obtained by the propensity score matching procedure, gastrectomy was a persistent significant protective predictor for overall survival (HR 0.51, $95 \%$ CI $0.47-0.56, P<0.001)$ and cancer-specific survival (HR $0.50,95 \%$ CI $0.46-0.55, P<0.001$ ). In addition, gastrectomy was confirmed as a protective predictor for overall survival (HR 0.50, $95 \%$ CI 0.46-0.55, 


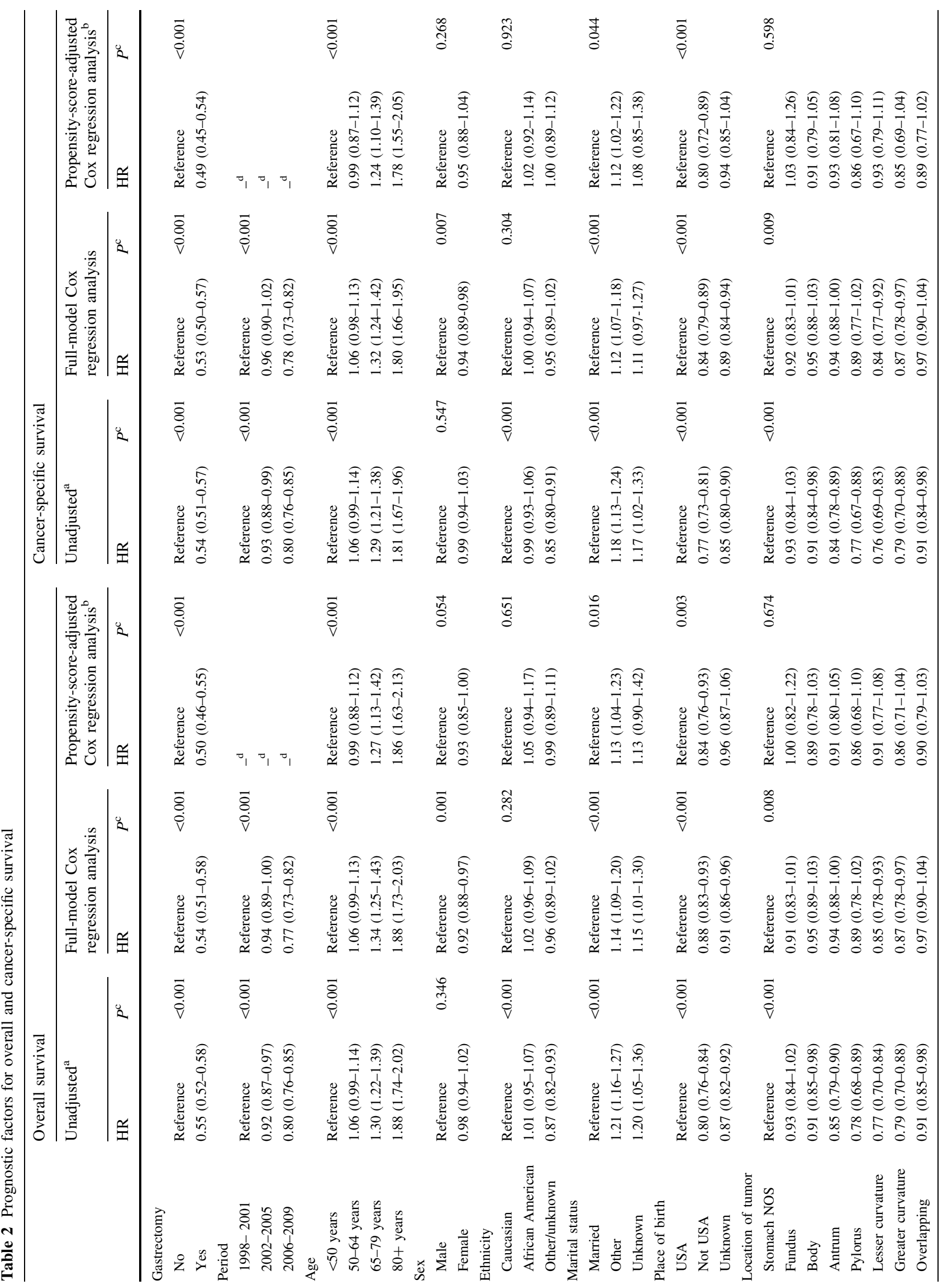




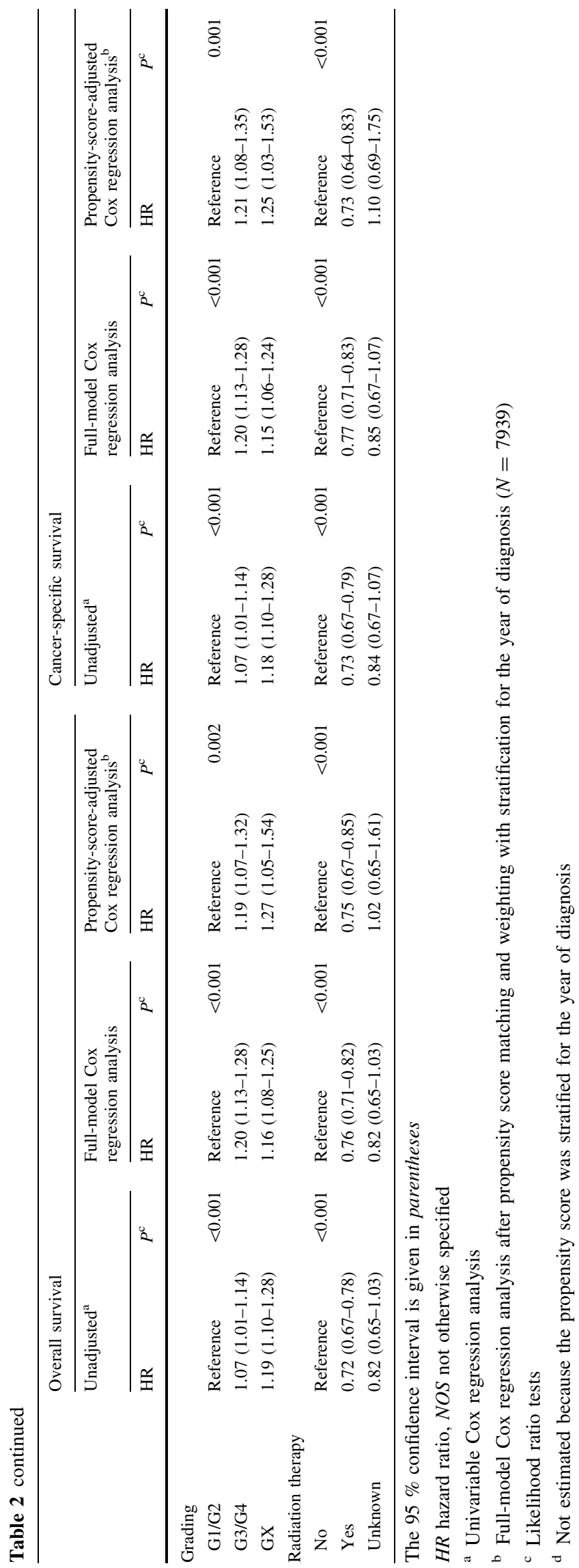

$P \leq 0.001)$ and cancer-specific survival (HR 0.49, $95 \%$ CI $0.45-0.54, P \leq 0.001$ ) in full-model Cox regression analysis, with use of the weights and strata obtained by the propensity score matching procedure and additionally adjustment for putative confounding variables (Table 2). In an auxiliary propensity score analysis based on inverse treatment probability weights, similar results were obtained, further confirming gastrectomy is associated with improved overall survival (HR 0.54, $95 \%$ CI $0.50-0.59$, $P \leq 0.001$ ) and cancer-specific survival (HR 0.54, $95 \%$ CI $0.49-0.58, P \leq 0.001$ ).

\section{Discussion}

The present study is, to our knowledge, the first populationbased analysis applying propensity score methods to determine the impact of primary tumor resection in metastatic gastric cancer patients on overall and cancer-specific survival. On the basis of this cohort of stage IV gastric cancer patients, identified from the SEER database between 1998 and 2009, the present investigation revealed the following results.

First, the rate of metastatic disease significantly increased over time. This is consistent with previous results [30] and might reflect the use of increasingly sensitive imaging modalities such as integrated PET/CT and the expanding use of staging laparoscopy. With that shift from the curative to the palliative setting thanks to modern diagnostic techniques, the question arises whether a dogmatic sectional view between palliative and curative therapeutic options is still constructive. Furthermore, one has to consider this shift as a potent factor influencing survival rates over time.

Second, the gastrectomy rate in patients with metastatic disease dropped mainly after 2006, which might be due to guideline recommendations, such as those of the European Society for Medical Oncology, not to operate on asymptomatic metastatic tumors and to new systemic therapeutic options [6, 31].

Third, the median survival in both groups is poor, and during 11-year period investigated, we observed no clinically relevant increase in median overall survival in patients who underwent palliative gastrectomy (1.5 months) and in those who did not undergo palliative gastrectomy (1 month).

Fourth, gastrectomy was associated with improved overall and cancer-specific survival, both in conventional Cox regression models and after propensity score matching.

These results are consistent with the results of two previous SEER-based analyses [20, 32]: Smith et al. [32] reported a significant survival advantage for patients with a 

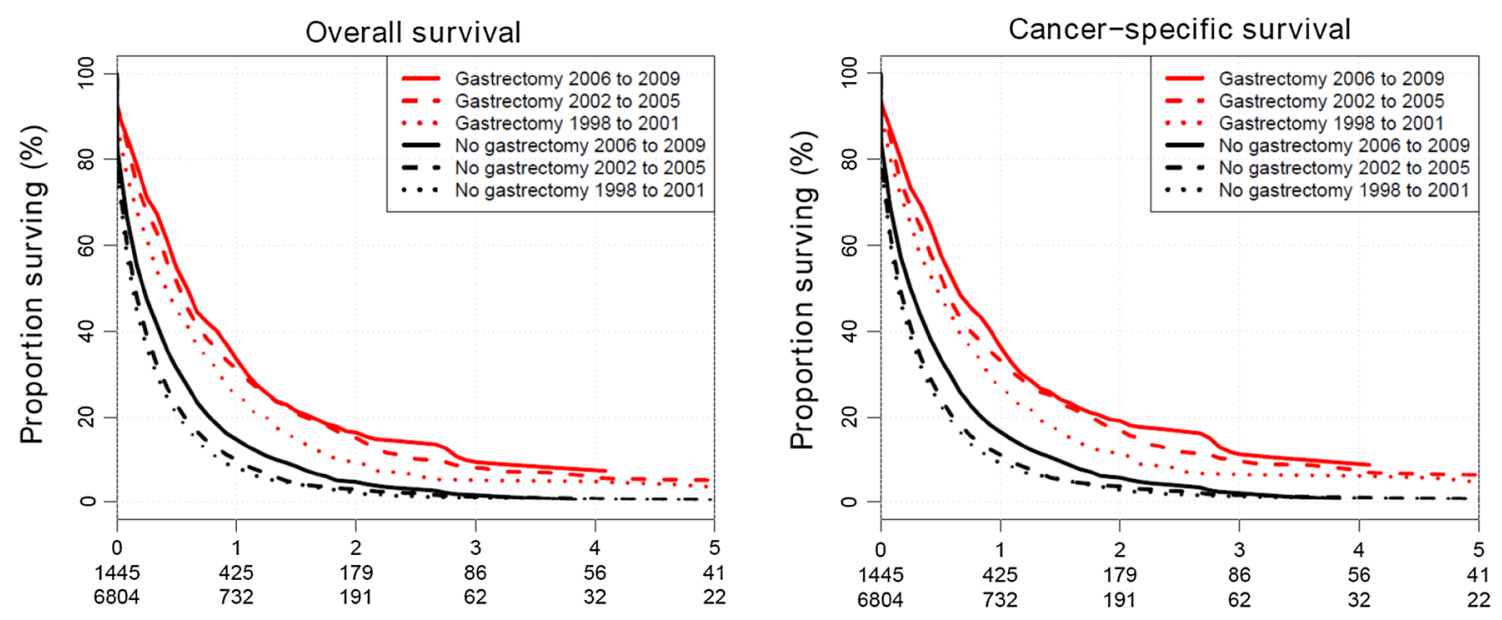

Years after diagnosis / Patients at risk with Gastrectomy | No gastrectomy

Years after diagnosis / Patients at risk with Gastrectomy | No gastrectomy
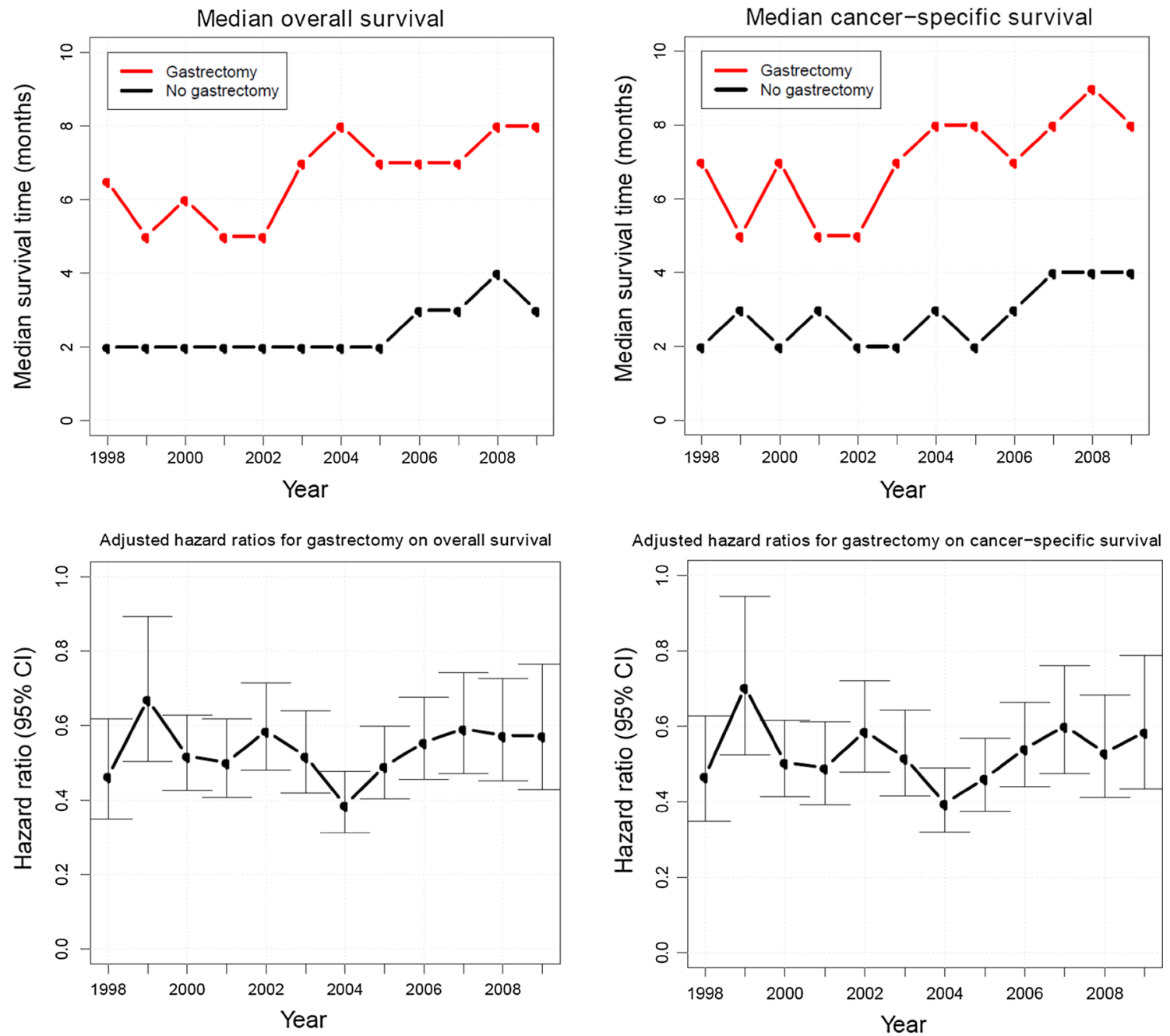

Fig. 3 Trend in overall and cancer-specific survival. $C I$ confidence interval

resected primary tumor from 1988 until 2005 (median overall survival of 3 months vs 9 months, $P<0.001$ ) with a hazard ratio of 0.5 . The present analysis confirmed these results even after optimal adjustment for potential confounders by propensity score matching. Also, the better survival rates in the gastrectomy group remain stable 
during the period from 1998 to 2008 regardless of new systemic therapeutic options (e.g., new combination chemotherapies or the addition of targeted therapy).

Propensity score analysis is becoming increasingly popular in medical research because this method, in principle, can effectively balance measured covariates across treatment groups in nonrandomized studies [24-26]. This method is named after and based on the estimation of a score for the propensity to receive a certain treatment conditional on baseline covariates [33]. In a second analytical step, the propensity score is incorporated in a statistical analysis assessing the outcome of interest. The term "propensity score matching" is used widely and covers four different main concepts. In pair matching, treated and untreated patients with similar propensity scores are paired. The other concepts are stratification, covariate adjustment, and inverse probability of treatment weighting. In the present study, two recent developments of the propensity score matching method were applied which combine these concepts. Full matching simultaneously combines matching, subclassification, and weighting [27]. The covariate balancing propensity score analysis is based on inverse treatment probability weights [34-37] and simultaneously adjusts the covariance structure [29]. Both methods confirmed gastrectomy to be associated with lower mortality. In a recent study on simulated data, full matching and inverse probability of treatment weighting were considered equivalent in most circumstances [38]. However, both propensity score methods used in this study share the general shortcomings of potential hidden bias due to unobserved or unrecorded variables [38]. Additionally, if the treatment groups relevantly differ regarding covariates, many individuals have to be excluded from the analysis, leading to a loss of power and generalizability. However, in the present study only 26 of 1445 patients who underwent gastrectomy and 266 of 6804 patients who did not undergo gastrectomy had to be excluded from the full-matching propensity score analysis. Hence, neither a loss of power nor generalizability seems to be a threat to the present analysis, especially when considering the population-based nature and the size of the patient cohort.

Since the results from randomized clinical trials concerning palliative gastrectomy in metastatic gastric cancer patients are still lacking (and most likely will never be available), the application of propensity score methods is probably the most appropriate method to assess the research question at hand. The advantage of the present analysis of the SEER database is the high power of a large cohort and the potential of the SEER database to mirror outcomes in the daily clinical routine.

However, the restrictions of the SEER database strongly limit the validity of the present investigation despite the use of propensity score methods. Since patients were not randomly assigned to the respective treatment group, selection bias is likely. Patients who underwent gastrectomy are likely to be highly selected, with fewer metastases and a better performance status compared with those who did not undergo gastrectomy. There are several relevant variables which are likely to impact the selection for or against palliative gastrectomy and the outcomes; however, they cannot be ascertained from the SEER registry. Indeed, data on the performance status, QoL, use of chemotherapy or targeted therapy, site of metastases, extension and number of metastases, resection margin status, and whether or not the primary tumor was symptomatic are lacking.

Overall, despite careful statistical adjustment, the probability of selection bias in the present analysis remains high. Clearly, patients with better prognosis to start with are likelier to undergo palliative resection. Other potential explanations beside a selection bias include hindering a further metastatic spread from the primary tumor, improved responsiveness to systemic therapies, and decreased immune suppression [39, 40].

However, metastatic gastric cancer is persistently a fatal disease with a low median survival of 7 months who underwent gastrectomy and 3 months for patients who did not undergo gastrectomy. There are only few mid-term survivors, with 3-year survival rates of 9.4 and $2.1 \%$ in patients who underwent palliative gastrectomy and who did not undergo palliative gastrectomy, respectively.

Considering the consistently low survival rate in metastatic gastric cancer over time, it is clear that improvement not only in survival but also in QoL is a key factor for decision-making. After palliative gastrectomy, QoL significantly decreases in the first three postoperative months [14]. Afterward, QoL gradually improves but does not fully recover within 1 year $[14,15]$. Hence, in terms of QoL, only midterm survivors are likely to benefit from palliative gastrectomy. Of note, however, QoL after palliative gastrectomy is better than after nonresectional gastrojejunostomy [9].

Recently, the results of a first, small randomized clinical trial (GYMSSA) of 17 patients which compared the impact of maximal cytoreductive surgery plus regional heated intraperitoneal chemotherapy plus chemotherapy versus chemotherapy alone have been published [41]. Its conclusion is that selected patients with gastric carcinomatosis and limited disease burden can achieve prolonged survival. The results from another randomized clinical trial (REGATTA) comparing gastrectomy plus chemotherapy with chemotherapy alone in metastatic gastric cancer patients are awaited [42].

In summary, we conclude that palliative resection of the primary tumor in metastatic gastric cancer patients is associated with improved overall and cancer-specific survival. However, considering the low survival rates with 
minor improvements over the past decade, only a minority of patients benefit from palliative gastrectomy. Palliative gastrectomy might be discussed in selected patients, particularly in those with symptoms and with the objective of improving QoL.

\section{Compliance with ethical standards}

For this type of study formal consent is not required. All procedures followed were in accordance with the ethical standards of the responsible committee on human experimentation (institutional and national) and with the Helsinki Declaration of 1964 and later versions.

Conflict of interest The authors declare that they have no conflict of interest.

\section{References}

1. Bertuccio P, Chatenoud L, Levi F, Praud D, Ferlay J, Negri E, et al. Recent patterns in gastric cancer: a global overview. Int $\mathrm{J}$ Cancer. 2009;125(3):666-73. doi:10.1002/ijc.24290.

2. Fock KM. Review article: the epidemiology and prevention of gastric cancer. Aliment Pharmacol Ther. 2014;40(3):250-60. doi:10.1111/apt.12814.

3. Dassen AE, Lemmens VE, van de Poll-Franse LV, Creemers GJ, Brenninkmeijer SJ, Lips DJ, et al. Trends in incidence, treatment and survival of gastric adenocarcinoma between 1990 and 2007: a population-based study in the Netherlands. Eur J Cancer. 2010;46(6):1101-10. doi:10.1016/j.ejca.2010.02.013.

4. Park SC, Chun HJ. Chemotherapy for advanced gastric cancer: review and update of current practices. Gut Liv. 2013;7(4):385-93. doi:10.5009/gnl.2013.7.4.385.

5. Wagner AD, Unverzagt S, Grothe W, Kleber G, Grothey A, Haerting J, et al. Chemotherapy for advanced gastric cancer. Cochrane Database Syst Rev. 2010;(3):CD004064. doi:10.1002/ 14651858.CD004064.pub3.

6. Waddell T, Verheij M, Allum W, Cunningham D, Cervantes A, Arnold D. Gastric cancer: ESMO-ESSO-ESTRO clinical practice guidelines for diagnosis, treatment and follow-up. Ann Oncol. 2013;24(Suppl 6):vi57-63. doi:10.1093/annonc/mdt344.

7. Mohri Y, Tanaka K, Ohi M, Saigusa S, Yasuda H, Toiyama Y, et al. Identification of prognostic factors and surgical indications for metastatic gastric cancer. BMC Cancer. 2014;14:409. doi:10. 1186/1471-2407-14-409.

8. Dittmar Y, Rauchfuss F, Goetz M, Jandt K, Scheuerlein H, Heise $\mathrm{M}$, et al. Non-curative gastric resection for patients with stage 4 gastric cancer-a single center experience and current review of literature. Langenbecks Arch Surg Dtsch Ges Chir. 2012;397(5):745-53. doi:10.1007/s00423-012-0902-3.

9. Samarasam I, Chandran BS, Sitaram V, Perakath B, Nair A, Mathew G. Palliative gastrectomy in advanced gastric cancer: is it worthwhile? ANZ J Surg. 2006;76(1-2):60-3. doi:10.1111/j. 1445-2197.2006.03649.x.

10. Sano T, Sasako M, Yamamoto S, Nashimoto A, Kurita A, Hiratsuka M, et al. Gastric cancer surgery: morbidity and mortality results from a prospective randomized controlled trial comparing D2 and extended para-aortic lymphadenectomy-Japan Clinical Oncology Group study 9501. J Clin Oncol. 2004;22(14):2767-73. doi:10.1200/jco.2004.10.184.

11. Collins A, Hatzaras I, Schmidt C, Carruthers K, Melvin WS, Muscarella P, et al. Gastrectomy in advanced gastric cancer effectively palliates symptoms and may improve survival in select patients. J Gastrointest Surg. 2014;18(3):491-6. doi:10. 1007/s11605-013-2415-y.

12. McCulloch P, Ward J, Tekkis PP. Mortality and morbidity in gastro-oesophageal cancer surgery: initial results of ASCOT multicentre prospective cohort study. BMJ. 2003;327(7425):1192-7. doi:10.1136/bmj.327.7425.1192.

13. Schwarz RE, Zagala-Nevarez K. Gastrectomy circumstances that influence early postoperative outcome. Hepatogastroenterology. 2002;49(48):1742-6.

14. Avery K, Hughes R, McNair A, Alderson D, Barham P, Blazeby J. Health-related quality of life and survival in the 2 years after surgery for gastric cancer. Eur J Surg Oncol. 2010;36(2):148-54. doi:10.1016/j.ejso.2009.09.008.

15. Kong H, Kwon OK, Yu W. Changes of quality of life after gastric cancer surgery. J Gastric Cancer. 2012;12(3):194-200. doi:10. 5230/jgc.2012.12.3.194.

16. Kahlke V, Bestmann B, Schmid A, Doniec JM, Kuchler T, Kremer B. Palliation of metastatic gastric cancer: impact of preoperative symptoms and the type of operation on survival and quality of life. World J Surg. 2004;28(4):369-75. doi:10.1007/ s00268-003-7119-0.

17. Value of palliative resection in gastric cancer. $\mathrm{Br} \mathrm{J}$ Surg. 2002;89(11):1438-43. doi:10.1046/j.1365-2168.2002.02220.x.

18. He MM, Zhang DS, Wang F, Wang ZQ, Luo HY, Jin Y, et al. The role of non-curative surgery in incurable, asymptomatic advanced gastric cancer. PLoS One. 2013;8(12):e83921. doi:10. 1371/journal.pone.0083921.

19. Lasithiotakis K, Antoniou SA, Antoniou GA, Kaklamanos I, Zoras O. Gastrectomy for stage IV gastric cancer. a systematic review and meta-analysis. Anticancer Res. 2014;34(5):2079-85.

20. Shridhar R, Dombi GW, Weber J, Hoffe SE, Meredith K, Konski A. Adjuvant radiation therapy increases overall survival in nodepositive gastric cancer patients with aggressive surgical resection and lymph node dissection: a SEER database analysis. Am J Clin Oncol. 2012;35(3):216-21. doi:10.1097/COC. 0b013e31820dbf08.

21. Ouchi K, Sugawara T, Ono H, Fujiya T, Kamiyama Y, Kakugawa $\mathrm{Y}$, et al. Therapeutic significance of palliative operations for gastric cancer for survival and quality of life. J Surg Oncol. 1998;69(1):41-4.

22. Gold JS, Jaques DP, Bentrem DJ, Shah MA, Tang LH, Brennan $\mathrm{MF}$, et al. Outcome of patients with known metastatic gastric cancer undergoing resection with therapeutic intent. Ann Surg Oncol. 2007;14(2):365-72. doi:10.1245/s10434-006-9059-z.

23. Surveillance, Epidemiology, and End Results Program. SEER research data (1973-2010), released April 2013, based on the November 2012 submission. National Cancer Institute, Division of Cancer Control and Population Sciences, Surveillance Research Program, Surveillance Systems Branch.

24. Rubin DB. Estimating causal effects from large data sets using propensity scores. Ann Intern Med. 1997;127(8 Pt 2):757-63.

25. Joffe MM, Rosenbaum PR. Invited commentary: propensity scores. Am J Epidemiol. 1999;150(4):327-33.

26. Rosenbaum PR. Model-based direct adjustment. J Am Stat Assoc. 1987;82(398):387-94. doi:10.1080/01621459.1987.10478441.

27. Hansen BB, Klopfer SO. Optimal full matching and related designs via network flows. J Comput Graph Stat. 2006;15:609-27.

28. Ho DE, Imai K, King G, Stuart EA. MatchIt: nonparametric preprocessing for parametric causal inference. J Stat Softw. 2011;42(8):1-28.

29. Imai K, Ratkovic M. Covariate balancing propensity score. J R Stat Soc B. 2014;76(1):243-63.

30. Rose JS, Bekaii-Saab TS. New developments in the treatment of metastatic gastric cancer: focus on trastuzumab. Onco Targets Ther. 2011;4:21-6. doi:10.2147/ott.s10188. 
31. Cunningham D. Gastric cancer: ESMO clinical recommendations for diagnosis, treatment and follow-up. Ann Oncol. 2007;18(Suppl 2):ii17-8. doi:10.1093/annonc/mdm019.

32. Smith JK, Hill JS, Ng SC, McDade TP, Shah SA, Tseng JF. Potential benefit of resection for stage IV gastric cancer: a national survey. J Gastrointest Surg. 2010;14(11):1660-8. doi:10. 1007/s11605-010-1351-3.

33. Rosenbaum PR, Rubin DB. The central role of the propensity score in observational studies for causal effects. Biometrika. 1983;70(1):41-55.

35. Hirano KI, Imbens GW, Ridder G. Efficient estimation of average treatment effects using the estimated propensity score. Econometrica. 2003;71(4):28.

34. Robins JM, Hernan MA, Brumback B. Marginal structural models and causal inference in epidemiology. Epidemiology. 2000;11(5):550-60.

36. Sato T, Matsuyama Y. Marginal structural models as a tool for standardization. Epidemiology. 2003;14(6):680-6. doi:10.1097/ 01.EDE.0000081989.82616.7d.

37. Hernan MA, Robins JM. Estimating causal effects from epidemiological data. J Epidemiol Community Health. 2006;60(7):578-86. doi:10.1136/jech.2004.029496.

38. Austin PC, Stuart EA. The performance of inverse probability of treatment weighting and full matching on the propensity score in the presence of model misspecification when estimating the effect of treatment on survival outcomes. Stat Methods Med Res. 2015. doi:10.1177/0962280215584401.

39. Fields RC, Jeffe DB, Trinkaus K, Zhang Q, Arthur C, Aft R, et al. Surgical resection of the primary tumor is associated with increased long-term survival in patients with stage IV breast cancer after controlling for site of metastasis. Ann Surg Oncol. 2007;14(12):3345-51. doi:10.1245/s10434-007-9527-0.

40. Fisher B, Gunduz N, Coyle J, Rudock C, Saffer E. Presence of a growth-stimulating factor in serum following primary tumor removal in mice. Cancer Res. 1989;49(8):1996-2001.

41. Rudloff U, Langan RC, Mullinax JE, Beane JD, Steinberg SM, Beresnev $\mathrm{T}$, et al. Impact of maximal cytoreductive surgery plus regional heated intraperitoneal chemotherapy (HIPEC) on outcome of patients with peritoneal carcinomatosis of gastric origin: results of the GYMSSA trial. J Surg Oncol. 2014;110(3):275-84. doi:10.1002/jso.23633.

42. Fujitani K, Yang HK, Kurokawa Y, Park do J, Tsujinaka T, Park $\mathrm{BJ}$, et al. Randomized controlled trial comparing gastrectomy plus chemotherapy with chemotherapy alone in advanced gastric cancer with a single non-curable factor: Japan Clinical Oncology Group study JCOG 0705 and Korea Gastric Cancer Association study KGCA01. Jpn J Clin Oncol. 2008;38(7):504-6. doi:10. 1093/jjco/hyn058. 